\title{
Prognostic signatures for renal cancer as identified by long non-coding and miRNA competing endogenous network analysis
}

\author{
HONGWEI GAO*, XUANRONG CHEN*, ZHIQUN SHANG and YUANJIE NIU \\ Department of Urology, Tianjin Institute of Urology, The Second Hospital of Tianjin Medical University, \\ Tianjin 300211, P.R. China
}

Received December 13, 2017; Accepted May 31, 2018

DOI: $10.3892 /$ or.2018.6476

\begin{abstract}
Long non-coding RNAs (lncRNAs) make up a significant portion of transcripts that are not translated into proteins and lncRNAs were previously thought to be transcriptional noise. Recently, there has been increased interest in lncRNAs due to their diverse functions in human cancers. At present, IncRNAs are considered to be the main component of the competing endogenous RNA (ceRNA) network due to their regulatory role in protein-coding gene expression by acting as microRNA (miRNA) sponges. However, the function of the IncRNA-mediated ceRNA network in renal cell carcinoma remains unknown. To examine the specific mechanism, we compared the expression profiles of mRNAs, lncRNAs, and miRNAs between renal cell carcinoma tissues and nontumor normal tissues using the Gene Expression Omnibus database. As a result, 5 lncRNAs were identified as aberrantly expressed and significantly correlated with the tumorigenesis and/or progression of renal cell carcinoma with the threshold value of absolute $\log _{2}$ fold change $>1$ and corrected $\mathrm{P}<0.05$. Among the 5 dysregulated lncRNAs, 2 of them were prognostic biomarkers according to the overall survival analysis for patients with renal cell carcinoma. We successfully constructed a dysregulated lncRNA-associated ceRNA network, which included 4 renal cell carcinoma-specific lncRNAs, 17 mRNAs and 2 miRNAs. In summary, the present study identified new lncRNA biomarkers and potential targets for renal cell carcinoma therapy. In particular, the novel ceRNA network identified in our study will be vital for understanding the
\end{abstract}

Correspondence to: Professor Yuanjie Niu, Department of Urology, Tianjin Institute of Urology, The Second Hospital of Tianjin Medical University, 23 Pingjiang Road, Hexi, Tianjin 300211, P.R. China

E-mail: yjniutjmu@outlook.com

*Contributed equally

Key words: renal cell carcinoma, lncRNA, ceRNA, miRNA, GEO regulatory mechanisms mediated by lncRNAs in the pathogenesis of renal cell carcinoma.

\section{Introduction}

Worldwide, renal cell carcinoma (RCC) accounts for $\sim 90 \%$ of all kidney neoplasms and 2-3\% of adult malignant tumors, and it is the ninth most common cancer (1-3). RCC leads to a considerable mortality rate, which is $\sim 100,000$ patients a year, and the morbidity and mortality rates are increasing at a rate of $2-3 \%$ per decade (4). There were $\sim 63,920$ newly diagnosed cases of RCC in the US in 2014 (5). The treatment of metastatic RCC has improved in the past few decades. Although cytoreductive nephrectomy is effective for the treatment of early and local RCC, $\sim 30 \%$ of patients suffer from metastatic disease after surgery. In addition, other therapeutic methods, such as immunotherapy have been used for the treatment of RCC; however, the effectiveness is limited (6). Therefore, the identification of potential biomarkers and therapeutic targets for RCC is urgently needed.

With the advancement of techniques used in genome-wide platforms, long non-coding RNAs (lncRNAs) have been identified and defined as RNA molecules of greater than 200 nucleotides in length and without apparent protein-coding potential $(7,8)$. Thousands of IncRNAs in both humans and mice have been identified by the combined analysis of Chip-seq and RNA-seq data; however, only a few have been assigned any function (9-11). Accumulating evidence suggests that lncRNAs play a vital role in various biological responses, such as the regulation of epigenetic silencing by chromatin remodeling, regulation of splicing, recruitment of transcription factors, and regulation of mRNA stability. IncRNAs can function by regulating the alternative splicing of pre-mRNAs and as competing endogenous RNAs (ceRNAs) that competitively bind to microRNAs (miRNAs) to suppress the inactivation of the target genes of miRNAs (12-14). Considering the potential significance of lncRNAs in physiological and pathological responses, many efforts have been made to elucidate the function of lncRNAs in carcinogenesis and cancer metastasis (15).

miRNAs are another class of non-coding RNAs with a length of $\sim 22$ nucleotides that have been extensively studied (16). miRNAs regulate the expression of target genes 
by partial complementary binding to miRNA response elements (MREs), which contain the corresponding mRNA sequences (17). One miRNA is able to regulate several mRNA transcripts, and one transcript may be the target of several different miRNAs. The miRNA regulatory network plays an important role in many biological responses, including carcinogenesis and tumor metastasis $(18,19)$. ceRNAs are transcripts that cross-regulate each other by competing for shared miRNAs $(20,21)$. mRNAs, lncRNAs and other RNAs can function as natural miRNA sponges by using shared MREs to suppress miRNA function (20). Certain lncRNAs have been identified and experimentally validated (22). H19 is an lncRNA that modulates the let-7 miRNA family members and miR-106a availability by acting as a molecular sponge $(23,24)$. The muscle-specific lncRNA linc-MD1 can interact with miR-133 to regulate the expression of muscle-specific gene expression (25). Such a RNA interaction could regulate many physiological and pathological responses and thus may provide new ideas for cancer therapy. Thus far, the IncRNAmiRNA-mRNA ceRNA network in hepatocellular, breast and gastric cancer, has been constructed and analyzed (26-29). However, similar studies involving RCC are rare. Moreover, genome-wide comprehensive analysis of RCC-associated miRNAs and lncRNAs has been limited.

In the present study, we analyzed the mRNA, IncRNA, and miRNA expression profiles from RCC-associated databases (GSE66270, GSE40914 and GSE71302, respectively). We identified a total of 2,378 aberrantly expressed RNAs; 2 upregulated and 3 downregulated RNAs were categorized as IncRNAs. Furthermore, we identified differentially expressed miRNAs (DEmiRNAs) in patients with RCC to elucidate the potential crosstalk between IncRNA, miRNA and mRNA. Ultimately, a lncRNA-miRNA-mRNA ceRNA network was constructed from 4 dysregulated lncRNAs, 17 mRNAs and 2 miRNAs by bioinformatic analysis.

\section{Materials and methods}

Study materials. Three RNA datasets (mRNA, IncRNA and miRNA) were selected in this study. In these datasets, GSE66270, GSE40914 and GSE71302 were analyzed as they were associated with renal cancer (30-32). GSE66270 (mRNA) of the Affymetrix Human Genome U133 Plus 2.0 Array (GPL570) platform consisted of 28 samples including 14 renal cancer and 14 normal samples. GSE40914 (lncRNA) was composed of three sub-datasets, GSE40911, GSE40912 and GSE40913. The GSE40911 dataset was selected for the extraction of differentially expressed lncRNAs (DElncRNAs). GSE40911 was based on the IQUSP_Human_intronic_4k_v2.0 (GPL3985) platform, which consisted of 44 samples including 22 renal cancer and 22 normal samples. GSE71302 (miRNA) was based on the Agilent-021827 Human miRNA Microarray (V3) (miRBase release 12.0 miRNA ID version) (GPL10850) platform, which consisted of 10 samples including 5 renal cancer and 5 normal samples.

Identification of differentially expressed genes. The analysis and extraction of differentially expressed genes were conducted with R language software, which included background correction, normalization, and calculation of the expression value of the original data and screening of the differentially expressed genes. The Benjamini-Hochberg (BH) method was used for statistical corrections. The online tool GEO2R was used for the extraction of differentially expressed genes as original data of the expression level for GSE40911 and GSE71302 were not available. In terms of the difference in expression threshold, genes were considered as significant with $\log _{2}$ FoldChangel $>1$ and corrected $\mathrm{P}<0.05$.

After all genes with different expression levels were collected, which were determined according to the previously set threshold for GSE40911, the corresponding GPL3985 platform was downloaded to annotate the data. The empty comment lines were deleted, and all the information of the non-coding RNAs were filtered out. Based on the information of the non-coding RNAs and all the differentially expressed genes, DElncRNAs were obtained.

Survival analysis. A total of 417 renal cancer samples in the database were retrieved from the Cancer Genome Atlas data portal. The survival package of $\mathrm{R}$ statistical programming software was used for the survival analysis of filtered DElncRNAs and DEmiRNAs.

Functional enrichment analysis. When a sufficient number of differentially expressed mRNAs (DEmRNAs) was obtained, enrichment analysis of the differentially expressed genes was carried out using the cluster Profile package of $\mathrm{R}$ software. The enrichKEGG and enrichGO functions were used for KEGG and Gene Ontology (GO) enrichment analysis, respectively. GO terms and KEGG pathways with $\mathrm{BH}$-corrected $\mathrm{P}<0.05$ were filtered out to obtain the final results. As for the pathway that ranked first in KEGG enrichment analysis and the pathway relevant to renal cancer, the Pathview package of R software was used for the mapping of related genes and determination of the location of differentially expressed genes in the entire pathway.

Construction of the ceRNA network and interactions among DEmRNAs. IncRNA-miRNA interactions and miRNA-mRNA interactions were predicted using miRcode (http://www. mircode.org/) and miRTarBase (http://mirtarbase.mbc.nctu. edu.tw/) $(33,34)$. The miRNA-mRNA pairs were validated by various methods including qRT-PCR, western blotting, ChIP-seq and microarray. To obtain a more reliable ceRNA network, high-correlation sets in the miRTarBase were selected to predict the interactions between miRNAs and mRNAs. The results of the lncRNA-miRNA-mRNA network were visualized by Cytoscape 3.3.0 (35).

STRING database was used to screen interactions among DEmRNAs with the threshold of combined score $>0.4$. The renal cancer sub-network was imported into the Cytoscape database to calculate the connectivity, and the top 10 genes with the highest degree were selected as hub genes.

Real-time quantitative PCR. Total RNA was isolated from ACHN and HK-2 cells using an RNeasy ${ }^{\circledR}$ Mini kit (Qiagen, Hilden, Germany) according to the manufacturer's instructions. First-strand cDNA was synthesized from $11 \mu \mathrm{g}$ of total RNA using the Transcript or First Strand cDNA Synthesis kit (Roche, Mannheim, Germany) as instructed by the 


\section{Normal Tumor}

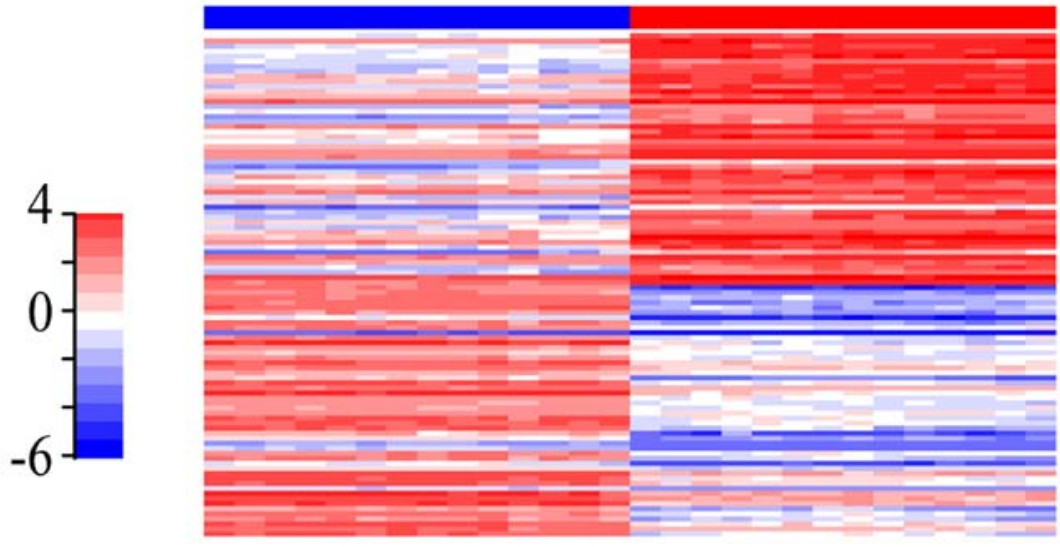

B

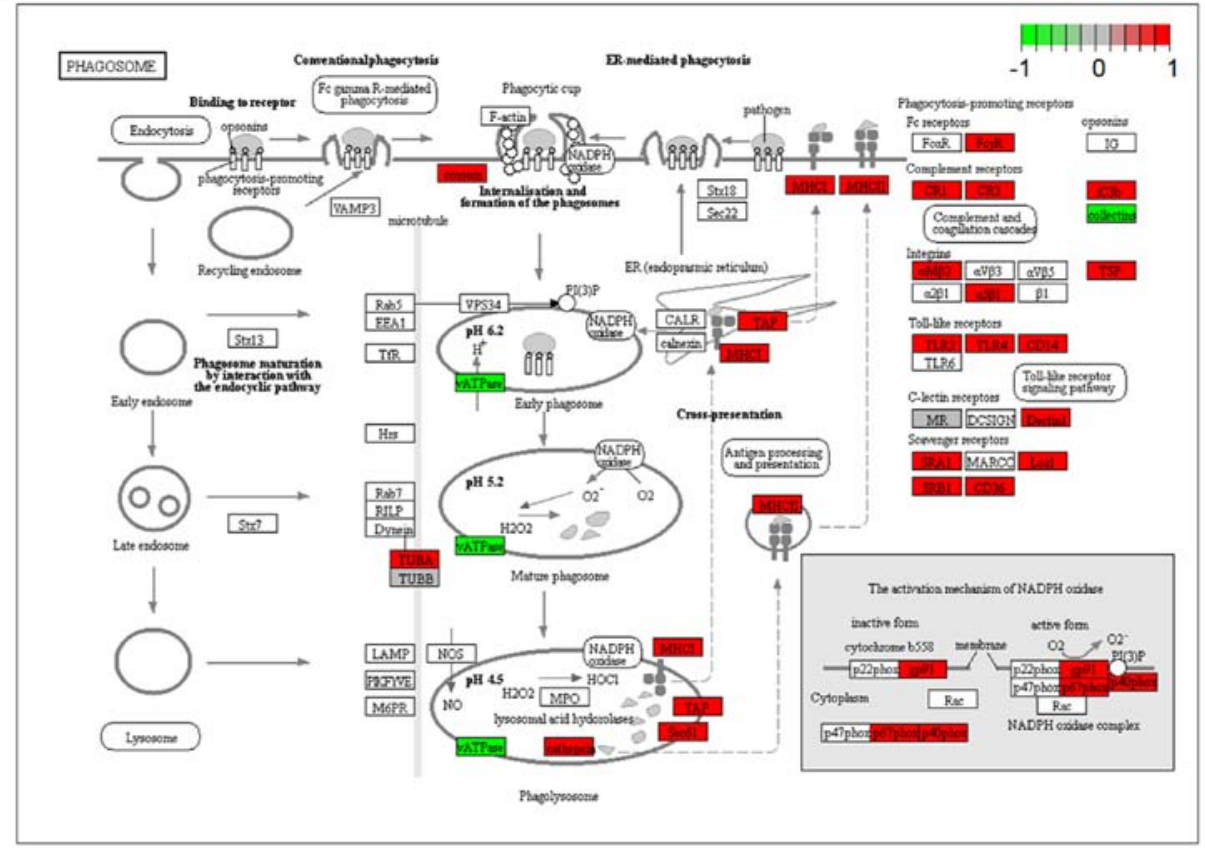

Figure 1. DEmRNAs in renal cancer. A total of 2,318 DEmRNAs were identified this study, and the relevant pathway analysis was performed using KEGG. (A) The expression profiles of the top 100 upregulated and 100 downregulated DEmRNAs were visualized using heatmaps. (B) Mapping of the phagosome pathway in the KEGG database. Red rectangles are upregulated DEmRNAs, and green boxes are downregulated DEmRNAs.

manufacturer. qRT-PCR reactions were performed on an Applied Biosystems ${ }^{\circledR} 7500$ real-time PCR system (Thermo Fisher Scientific, Inc., Waltham, MA, USA) using the following procedure: $95^{\circ} \mathrm{C}$ for $10 \mathrm{~min}$, followed by 40 cycles of $95^{\circ} \mathrm{C}$ for $30 \mathrm{sec}$ and $60^{\circ} \mathrm{C}$ for $1 \mathrm{~min}$. To create a qRT-PCR standard, glyceraldehyde 3-phosphate dehydrogenase (GAPDH) was used as an internal control. The $2^{-\Delta \Delta \mathrm{Ct}}$ method was used for data analysis.

\section{Results}

DEmRNAs and enrichment analysis in renal cancer. According to the threshold value of absolute $\log _{2}$ fold change $>1$ and corrected $\mathrm{P}<0.05,2,318$ mRNAs including 1,370 upregulated and 948 downregulated genes were identified as DEmRNAs in the GSE66270 database. The top 100 upregulated and the top 100 downregulated genes were outlined through the heatmap function in the gplots package of R software (Fig. 1A). The results demonstrated that the expression of selected RNAs was significantly different between renal cancer and normal tissues.

For a better understanding of the mechanisms of these differentially expressed genes, which were implicated in the tumorigenesis of renal cancer, functional enrichment analysis was performed using the enrichKEGG function of $\mathrm{R}$ software based on KOBAS 2.0. The phagosome pathway was identified as the pathway most significantly related to cancer $(\mathrm{P}<0.01)$; thus, visualization of the phagosome pathway was performed using the Pathview package. As shown in Fig. 1B, 37 DEmRNAs (33 upregulated and 4 downregulated) were involved in the phagosome pathway. Furthermore, the mapping of hsa05211 (the RCC pathway), associated with the tumorigenesis of renal cancer, was also performed. As shown in Fig. 1C, 15 DEmRNAs (12 upregulated and 3 downregulated) were 


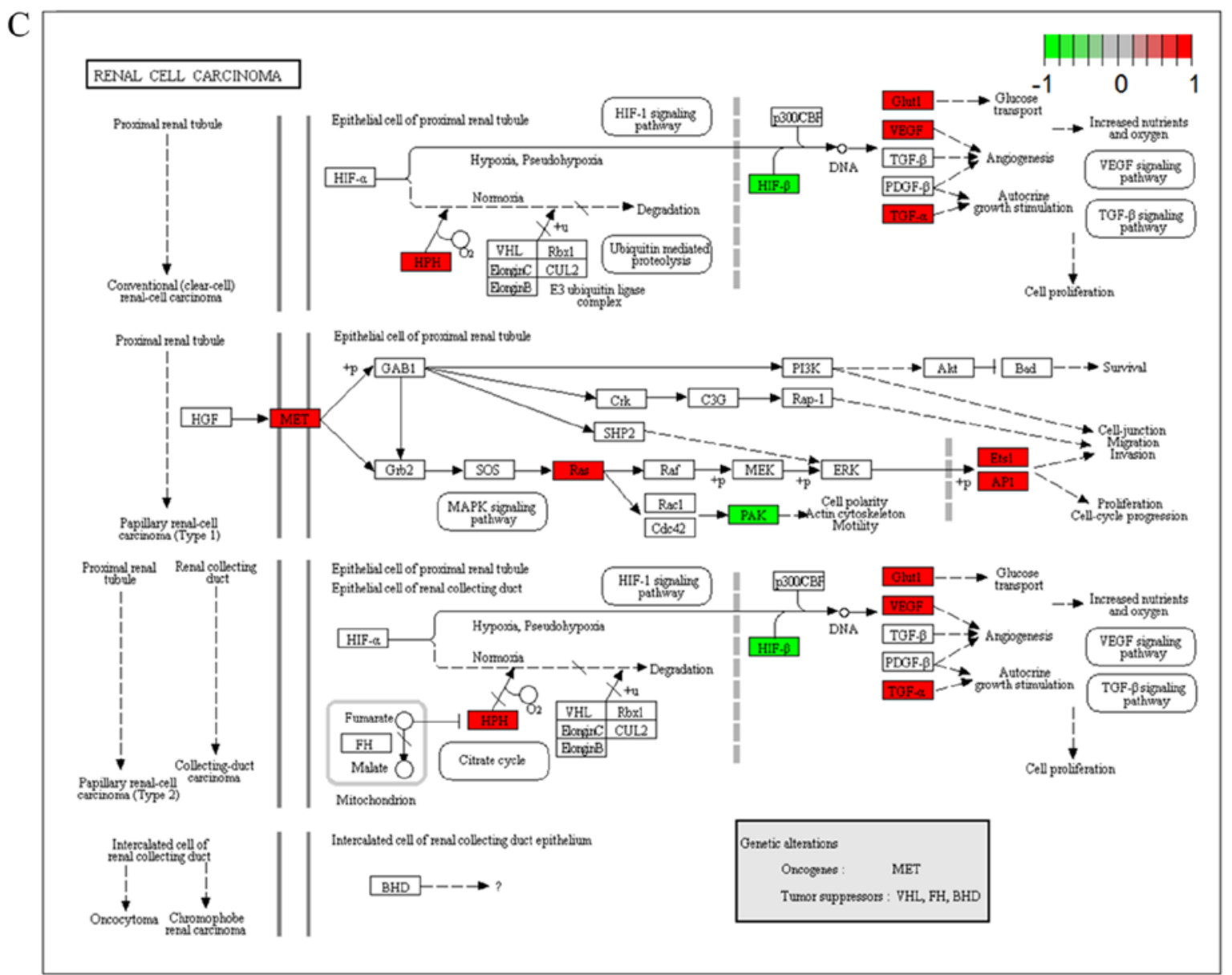

Figure 1. Continued. (C) Mapping of the hsa05211 (renal cell carcinoma) pathway in the KEGG database. Red boxes are upregulated DEmRNAs, and green boxes are downregulated DEmRNAs. DEmRNAs, differentially expressed miRNAs.
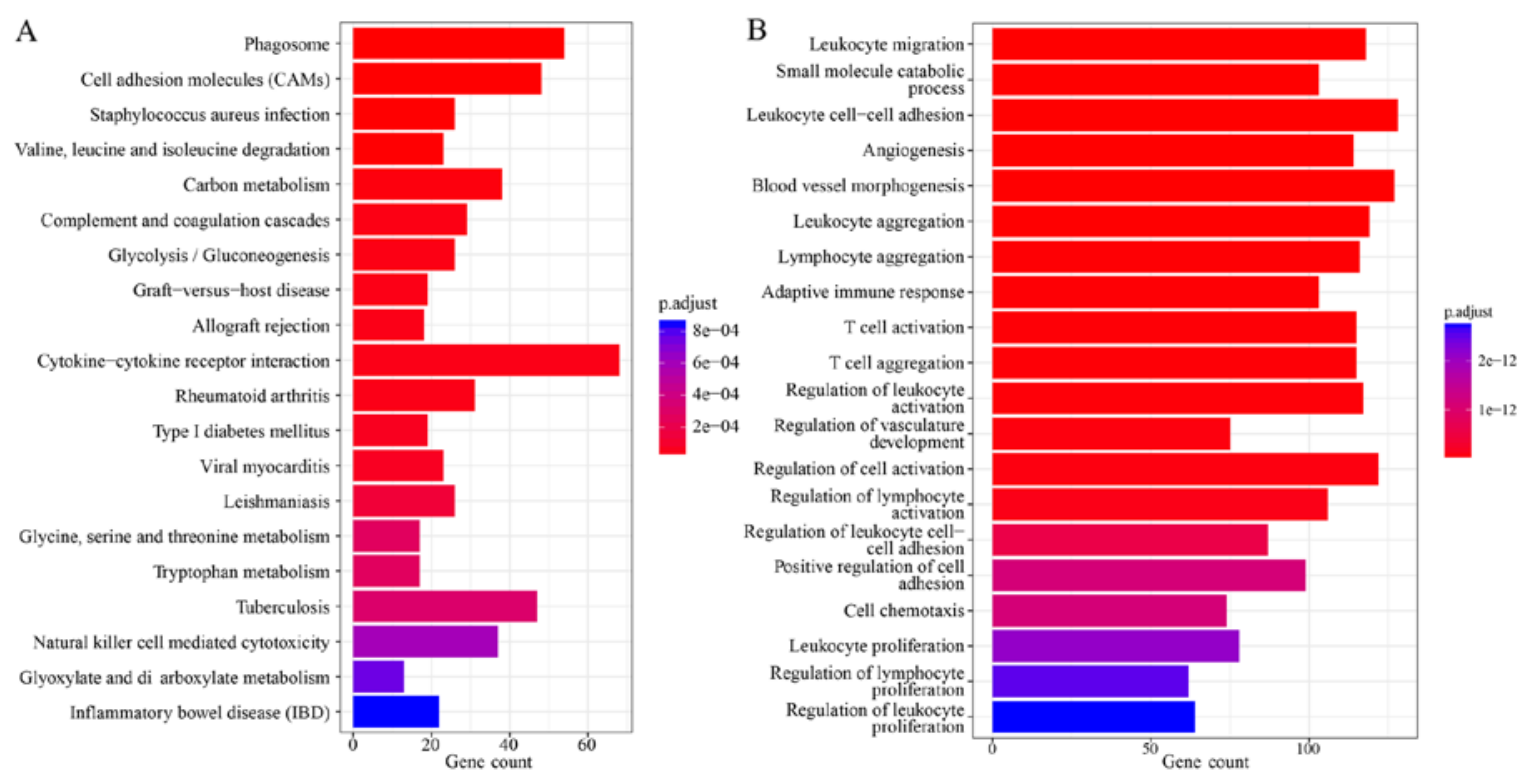

Figure 2. Functional enrichment analysis of DEmRNAs. (A) The top 20 most significantly enriched GO terms of DEmRNAs. (B) The top 20 most significantly enriched KEGG pathways of DEmRNAs. DEmRNAs, differentially expressed miRNAs.

involved in the hsa05211 pathway. In addition, Fig. 2 shows the top 20 significantly enriched GO terms (Fig. 2A) and KEGG pathways (Fig. 2B) of DEmRNAs.
DElncRNAs and DEmiRNAs in renal cancer. According to the threshold value of absolute $\log _{2}$ fold change $>1$ and corrected $\mathrm{P}<0.05,5$ lncRNAs, which included 2 upregulated 
Table I. Differentially expressed lncRNAs in renal cancer.

\begin{tabular}{llll}
\hline GENE & $\operatorname{logFC}$ & adj.P.Val & P-value \\
\hline ACTN4 & 1.005228 & $6.45 \mathrm{E}-08$ & $1.43 \mathrm{E}-09$ \\
CTHRC1 & -2.63837 & $1.56 \mathrm{E}-12$ & $7.13 \mathrm{E}-15$ \\
IGFBP7 & -2.29773 & $4.46 \mathrm{E}-10$ & $5.25 \mathrm{E}-12$ \\
RAB31 & -1.199 & $2.86 \mathrm{E}-10$ & $3.27 \mathrm{E}-12$ \\
RBPMS & 1.296871 & $1.56 \mathrm{E}-09$ & $1.99 \mathrm{E}-11$ \\
\hline
\end{tabular}

lncRNAs, long non-coding RNAs; FC, fold change; ACTN4, actinin alpha 4; CTHRC1, collagen triple helix repeat containing 1; IGFBP7, insulin like growth factor binding protein 7; $R A B 31, \mathrm{RAB} 31$, member RAS oncogene family; RBPMS, RNA binding protein, mRNA processing factor.

and 3 downregulated lncRNAs (Table I), were identified as differentially expressed in the GSE40911 database. In addition, 55 miRNAs, which included 28 upregulated and 27 downregulated miRNAs, were identified as differentially expressed in the GSE71302 database.

The ceRNA network in renal cancer. For a better understanding of the characteristics of DElncRNAs in renal cancer, a dysregulated IncRNA-miRNA-mRNA ceRNA network was constructed by extracting the interaction pairs of IncRNAmiRNA in miRcode and miRNA-mRNA in miRTarBase. In the ceRNA network, 17 DEmRNAs (14 upregulated and 3 downregulated) and 5 DElncRNAs (2 upregulated and 3 downregulated) were identified. Moreover, 2 upregulated miRNAs (hsa-miR-570 and hsa-miR-508-3p) were identified in the network. The ceRNA network was divided into two parts, and the miRNA was located at the center of each part. The two parts were connected through 2 lncRNAs, which included the upregulated RBPMS and the downregulated RAB31. The network is shown in Fig. 3A.

Furthermore, the DEmRNAs involved in the ceRNA network were analyzed by KOBAS 3.0 to uncover signaling pathways that are regulated by DElncRNAs indirectly. As shown in Table II, 6 KEGG pathways were significantly enriched $(\mathrm{P}<0.05)$, and the top KEGG pathway was the diabetic complications pathway. Moreover, the other top 2 KEGG pathways were renal cancer-related pathways.
Table III. The top 10 DEmRNAs with highest degree.

\begin{tabular}{llc}
\hline Gene symbol & \multicolumn{1}{c}{ Gene name } & Degree \\
\hline FN1 & Fibronectin 1 & 98 \\
FBXO6 & F-box protein 6 & 82 \\
TP53 & Tumor protein P53 & 72 \\
MYC & MYC proto-oncogene & 66 \\
CDK1 & Cyclin-dependent kinase 1 & 62 \\
$J U N$ & Jun proto-oncogene & 47 \\
LYN & LYN proto-oncogene & 47 \\
SHC1 & SHC adaptor protein 1 & 46 \\
$L C K$ & LCK proto-oncogene & 45 \\
$I T G A 4$ & Integrin subunit $\alpha 4$ & 42 \\
\hline
\end{tabular}

It is possible that DElncRNAs could indirectly interact with DEmRNAs in renal cancer through miRNAs, such as hsa-miR-570 and hsa-miR-508-3p, as demonstrated in the ceRNA network. To explore the co-expression of each RNA in the network, the heatmaps of the expression profiles of DElncRNAs, DEmRNAs and DEmiRNAs were obtained (Fig. 3B).

Survival analysis of lncRNAs. The overall survival analysis of the 5 DElncRNAs in patients with renal cancer was performed by Kaplan-Meier curve analysis. As shown in Fig. 4, the expression levels of RAB31 ( $\mathrm{HR}=1.017$, 95\% CI, 0.971-1.432) and ACTN4 (HR=1.130, 95\% CI, 1.011-1.659) were negatively correlated with the overall survival of patients $(\mathrm{P}<0.05)$. Survival analysis of the DEmiRNAs showed that 3 highly expressed DEmiRNAs were negatively correlated with the patient overall survival, which were miR-362-5p ( HR=1.113, 95\% CI, 1.017-1.590), miR-508-3p $(\mathrm{HR}=0.830,95 \% \mathrm{CI}, 0.613-0.932)$, and $\mathrm{miR}-$ $532-5 p(H R=0.787,95 \% \mathrm{CI}, 0.548-0.916)$. The results are presented in Fig. 5.

Screening of key genes. According to the threshold of combined score $>0.4$, a total of 3,277 interaction pairs among the DEmRNAs were obtained. The top 10 genes with the highest degree are shown in Table III.

Table II. KEGG pathways of DEmRNAs that were involved in the ceRNA network.

\begin{tabular}{llr}
\hline ID & \multicolumn{1}{c}{ Pathway category } & P-value \\
\hline hsa04933 & AGE-RAGE signaling pathway in diabetic complications & $7.78 \mathrm{E}-04$ \\
hsa05211 & Renal cell carcinoma & $2.70 \mathrm{E}-02$ \\
hsa04610 & Complement and coagulation cascades & $3.17 \mathrm{E}-02$ \\
hsa04066 & HIF-1 signaling pathway & $4.10 \mathrm{E}-02$ \\
hsa04668 & TNF signaling pathway & $4.37 \mathrm{E}-02$ \\
hsa04142 & Lysosome & $4.87 \mathrm{E}-02$ \\
\hline
\end{tabular}

DEmRNAs, differentially expressed miRNAs; ceRNA, competing endogenous RNA. 

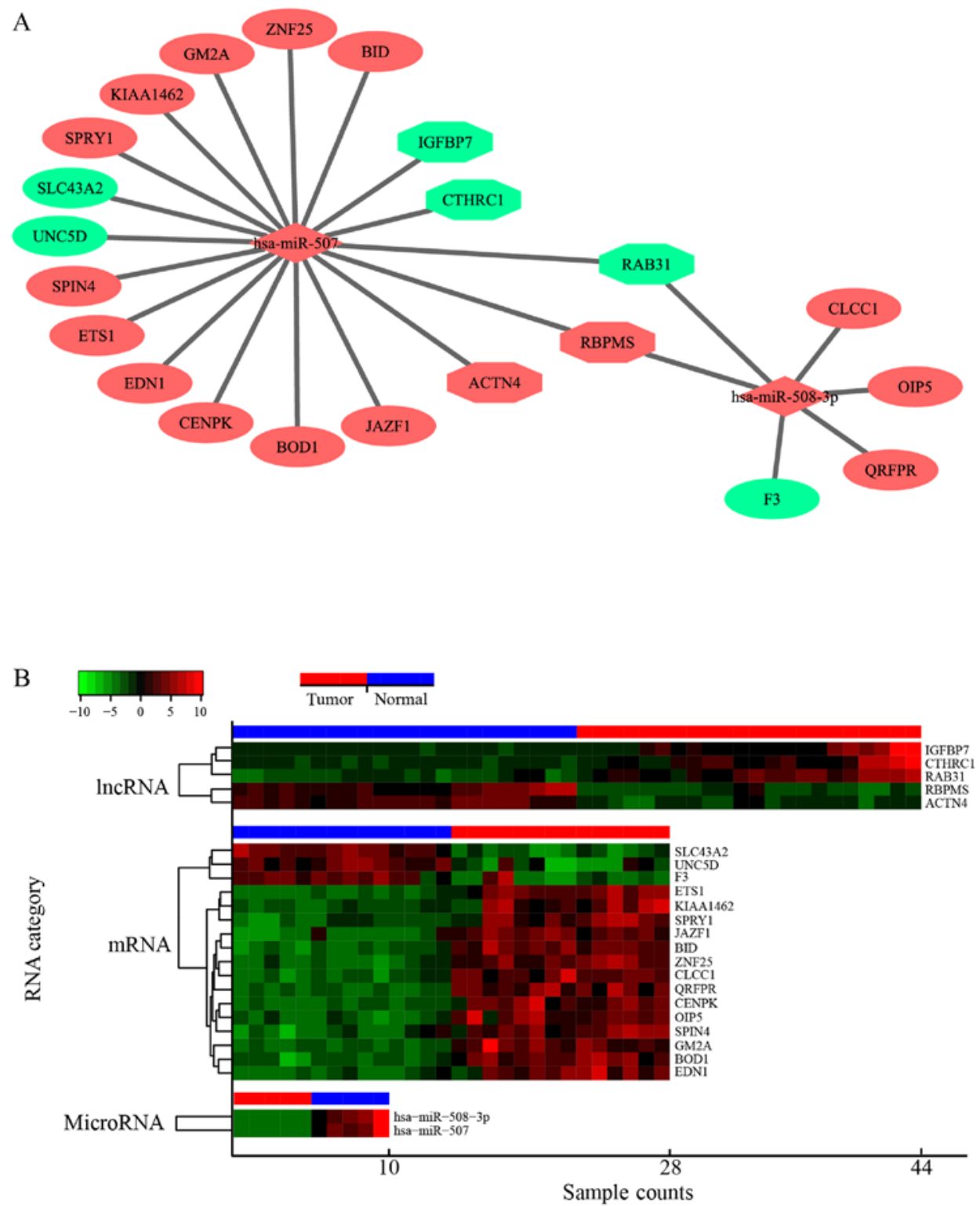

Figure 3. The ceRNA network mediated by DElncRNAs in renal cancer. (A) Global view of the IncRNA-miRNA-mRNA network in renal cancer. Upregulated genes are indicated in red color, and downregulated genes are indicated in green color. IncRNAs, miRNAs, and mRNAs are represented as hexagon, rhombus, and ellipse, respectively. (B) Heatmap of the expression profiles of lncRNAs, miRNAs and mRNAs in the network. The horizontal axis represents the sample capacity and the vertical axis represents the types of RNA. The blue and red bars at the top of the heatmap represent normal and renal cancer samples, respectively. ceRNA, competing endogenous RNA; DEmRNAs, differentially expressed miRNAs.

RAB31

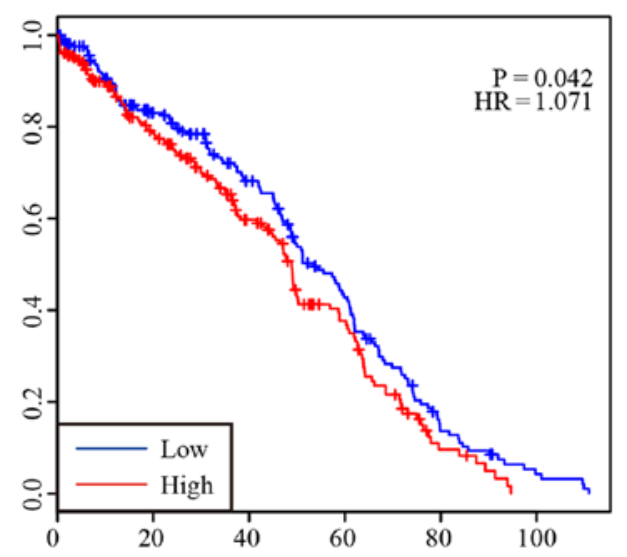

ACTN4

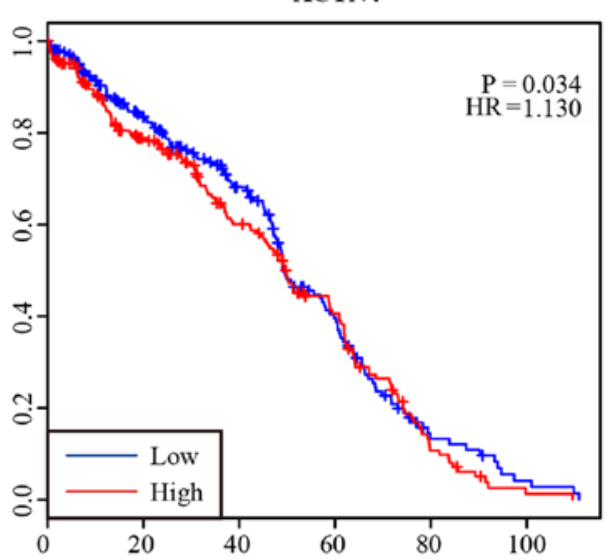

Figure 4. Kaplan-Meier analysis of the RAB31 and ACTN4 lncRNAs to determine the overall survival of patients with renal cancer. ACTN4, actinin alpha 4; $R A B 31, \mathrm{RAB} 31$, member RAS oncogene family. $\mathrm{x}$-axis and $\mathrm{y}$-axis represents survival time in month and survival probability. 

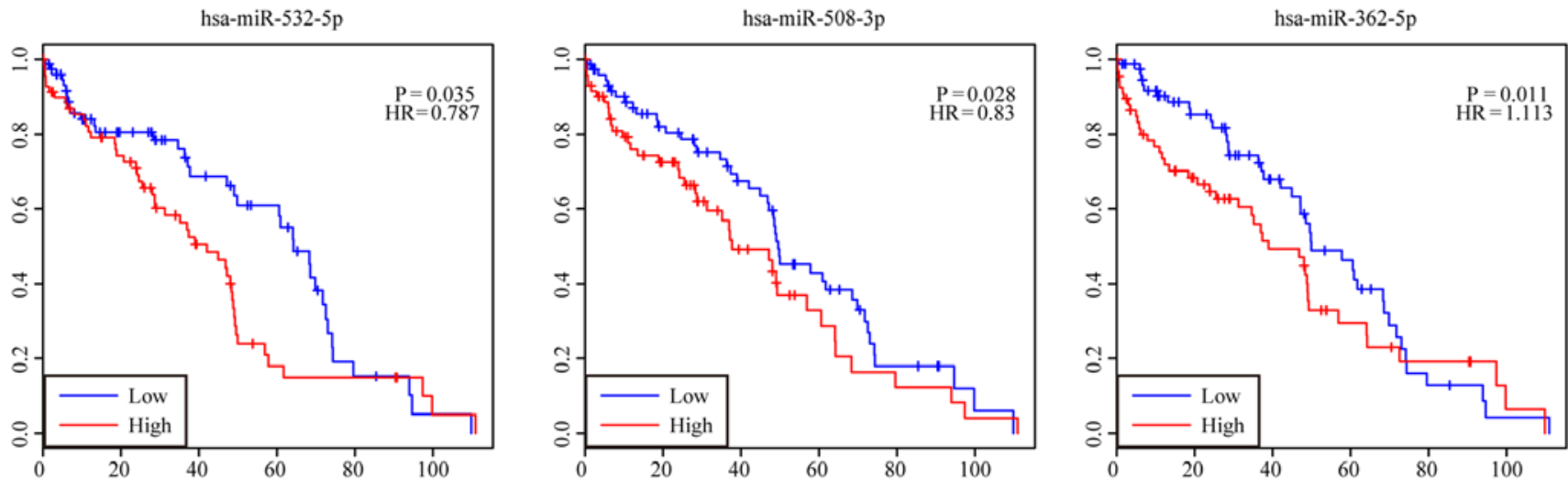

Figure 5. Kaplan-Meier analysis of the hsa-miR-532-5p, hsa-miR-508-3p and hsa-miR-362-5p miRNAs to determine the overall survival of patients with renal cancer. $\mathrm{x}$-axis and $\mathrm{y}$-axis represents survival time in month and survival probability.

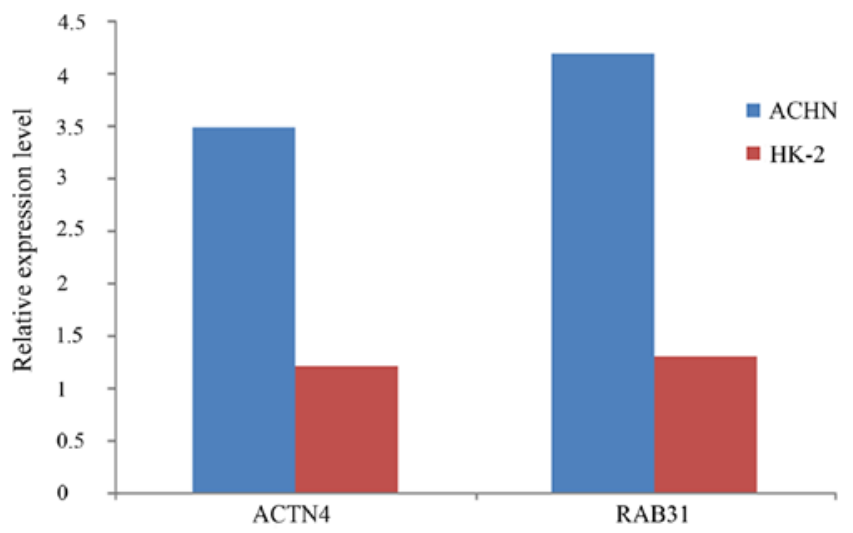

Figure 6. qRT-PCR analysis of ACTN4 and RAB31 in ACHN RCC cells and HK-2 normal renal cells. ACTN4, actinin alpha 4; RAB31, RAB31, member RAS oncogene family.

Real-time quantitative PCR. Expression differences of ACTN4 and RAB31 between RCC cells ACHN and normal renal cells HK-2 were explored thorough qRT-PCR. Primers used for ACTN4 were $\left(5^{\prime} \rightarrow 3^{\prime}\right)$ : forward primer, 5-ATGGTG GACTACCACGCGGCGAACC-3 and reverse primer, 5-TCA CAGGTCGCTCTCGCCATACAAG-3; primers used for RAB31 were $\left(5^{\prime} \rightarrow 3^{\prime}\right)$ : forward primer, 5 -ATGATGGCGATA CGGGAGCTCAAAG-3 and reverse primer, 5-TCAACA GCACCGGCGGCTGGCTTGC-3. Consistent with the results from the microarray analysis, both RAB31 and ACTN4 were upregulated in RCC cells compared with levels in the normal cells (Fig. 6).

\section{Discussion}

Increasing evidence suggests that lncRNAs are crucial in human cancer. Various studies have revealed that the differential expression of IncRNAs plays an important role in the development of cancer $(36,37)$. Recently, there have been efforts to identify aberrantly expressed lncRNAs in muscleinvasive bladder cancer. Based on a large sample size from the TCGA data portal, several dysregulated IncRNAs have been identified (38). However, a limited number of studies have identified RCC-specific IncRNAs.
Currently, lncRNAs closely associated with tumor status are thought to be more suitable than mRNAs as diagnostic and prognostic biomarkers (39). Some IncRNAs that have been well studied, such as H19, are considered as powerful predictors or potential targets in cancers (40). However, the relationship of IncRNAs with RCC remains unclear. Therefore, the expression profiles of lncRNAs, miRNAs and mRNAs in RCC were analyzed with a focus on the clinical diagnostic significance of DElncRNAs. In the present study, 5 lncRNAs with aberrant expression were identified in RCC compared with normal samples. In addition, 2 DElncRNAs (ACTN4 and RAB31) were included in the ceRNA network, which were significantly correlated with the overall survival of patients with RCC; this result strongly indicated that these DElncRNAs may function not only as key oncogenes but also as prognostic markers in RCC progression.

ACTN4 is a non-muscle type $\alpha$-catenin that is only expressed in non-muscle cells (41). In particular, ACTN4 is associated with cell migration and cell adhesion, and it was first identified in 1988 as a metastasis-related gene (42). In this study, the expression of ACTN4 was negatively correlated with overall survival, which was consistent with the results of a previous study reporting that patients with ACTN4 amplification have significantly worse overall survival in comparison with those without ACTN4 amplification (43). Various studies have shown that ACTN4 is expressed in several types of cancers, such as invasive ductal adenocarcinoma and ovarian cancer, and it has been used as a biomarker for therapeutic response $(41,43,44)$. RAB31 belongs to the Ras superfamily of small GTPase and was first identified in human melanocytes $(45,46)$. There has been increased interest in the relationship between RAB31 and human cancer. Gene expression profiling analysis has revealed the overexpression of RAB31 in estrogen receptor $\alpha$-positive breast carcinomas (47). Elevated transcript levels of RAB31 were reported in breast cancer cells expressing the urokinase-type plasminogen activator (uPA)-receptor splice variant uPAR-del4/5, and high Rab31 levels were found to be significantly associated with overall survival and distant metastasis-free survival (48-50). A meta-analysis of microarray data found that Rab31 is one of the 10 key genes in glioblastoma multiforme development (51). In this study, our results showed that the expression of RAB31 
was negatively associated with the overall survival of patients, which was consistent with the previously reported results for many types of cancers. These lncRNAs were identified as prognostic predictors, and they may contribute to the targeted therapy of RCC in the future.

Several miRNAs have been well characterized in human diseases including lung cancer and bladder cancer $(52,53)$. However, only a few IncRNAs have been mechanically and functionally characterized. In this study, we identified specific lncRNAs and miRNAs of RCC in a database and constructed a ceRNA network, which would be relevant for further investigations. Many protein-coding genes in the ceRNA network, such as FN1, TP53 and MYC, are known as oncogenes and/or tumor inhibitors related to RCC development and progression, which may be potential therapeutic targets of cancer (54-56). Six pathways of DEmRNAs were significantly enriched in the ceRNA network, and the top KEGG pathway was the diabetic complications pathway. This finding indicated that the diabetic complications pathway may be involved in the progression of RCC.

Although there has been increased interest in the function of IncRNAs in recent years, the functional association of lncRNAs and miRNAs remains unclear. The identification of DEmiRNAs in RCC is necessary for determining the oncogenic pathways mediated by miRNA. In this study, we identified miRNAs related to tumor initiation in renal cells. Moreover, a IncRNA-miRNA-mRNA ceRNA network was constructed to illustrate the interaction between miRNAs, lncRNAs and coding genes. Two DElncRNAs (ACTN4 and RAB31) were identified in this study, which may be potential prognostic markers and oncogenes in RCC progression. In particular, the ceRNA network built in this study would contribute to the elucidation of the unknown ceRNA regulatory network in RCC.

In conclusion, we identified tumor initiation-related miRNAs, and the IncRNA-miRNA-mRNA ceRNA network in RCC was constructed. The interaction between miRNAs, lncRNAs and coding genes was investigated. Further studies would help to elucidate the primary mechanism of miRNAs and lncRNAs in RCC.

\section{Acknowledgements}

Not applicable.

\section{Funding}

The present study was supported by the National Natural Science Foundation of China (grant nos.81472682 and 81772756) and the Natural Science Foundation of Tianjin (nos. 17JCZDJC35300, 15JCZDJC35400 and 15JCYBJC27200).

\section{Availability of data and materials}

The datasets used during the present study are available from the corresponding author upon reasonable request.

\section{Authors' contributions}

HWG and XRC put forward the ideas of the research, analyzed the data and written the manuscript. ZQS put forward the ideas of the article and helped revising the manuscript. YJN provided valuable instructions and suggestions on this thesis and helped revising the manuscript. All authors read and approved the manuscript and agree to be accountable for all aspects of the research in ensuring that the accuracy or integrity of any part of the work are appropriately investigated and resolved.

\section{Ethics approval and consent to participate}

Not applicable.

\section{Patient consent for publication}

Not applicable.

\section{Competing interests}

The authors declare that they have no competing interests.

\section{References}

1. Jonasch E, Gao J and Rathmell WK: Renal cell carcinoma. BMJ 349: g4797, 2014.

2. Ljungberg B, Campbell SC, Choi HY, Jacqmin D, Lee JE, Weikert S and Kiemeney LA: The epidemiology of renal cell carcinoma. Eur Urol 60: 615-621, 2011.

3. Gupta S, Kang HC, Ganeshan DM, Bathala TK and Kundra V: Diagnostic approach to hereditary renal cell carcinoma. AJR Am J Roentgenol 204: 1031-1041, 2015.

4. Bex A, Jonasch E, Kirkali Z, Mejean A, Mulders P, Oudard S, Patard JJ, Powles T, van Poppel H and Wood CG: Integrating surgery with targeted therapies for renal cell carcinoma: Current evidence and ongoing trials. Eur Urol 58: 819-828, 2010.

5. Siegel R, Ma J, Zou Z and Jemal A: Cancer statistics, 2014. CA Cancer J Clin 64: 9-29, 2014.

6. Wyczólkowski M, Klima W, Bieda W and Walas K: Spontaneous regression of hepatic metastases after nephrectomy and metastasectomy of renal cell carcinoma. Urol Int 66: 119-120, 2001.

7. International Human Genome Sequencing Consortium: Finishing the euchromatic sequence of the human genome. Nature 431: 931-945, 2004

8. Bergmann JH and Spector DL: Long non-coding RNAs: Modulators of nuclear structure and function. Curr Opin Cell Biol 26: 10-18, 2014.

9. Guttman M, Garber M, Levin JZ, Donaghey J, Robinson J, Adiconis X, Fan L, Koziol MJ, Gnirke A, Nusbaum C, et al: $\mathrm{Ab}$ initio reconstruction of cell type-specific transcriptomes in mouse reveals the conserved multi-exonic structure of lincRNAs. Nat Biotechnol 28: 503-510, 2010.

10. Cabili MN, Trapnell C, Goff L, Koziol M, Tazon-Vega B, Regev A and Rinn JL: Integrative annotation of human large intergenic noncoding RNAs reveals global properties and specific subclasses. Genes Dev 25: 1915-1927, 2011.

11. Guttman M, Amit I, Garber M, French C, Lin MF, Feldser D, Huarte M, Zuk O, Carey BW, Cassady JP, et al: Chromatin signature reveals over a thousand highly conserved large non-coding RNAs in mammals. Nature 458: 223-227, 2009.

12. Geisler S and Coller J: RNA in unexpected places: Long noncoding RNA functions in diverse cellular contexts. Nat Rev Mol Cell Biol 14: 699-712, 2013.

13. Tripathi V, Shen Z, Chakraborty A, Giri S, Freier SM, Wu X, Zhang Y, Gorospe M, Prasanth SG, Lal A and Prasanth KV: Long noncoding RNA MALAT1 controls cell cycle progression by regulating the expression of oncogenic transcription factor B-MYB. PLoS Genet 9: e1003368, 2013.

14. Tay Y, Rinn J and Pandolfi PP: The multilayered complexity of ceRNA crosstalk and competition. Nature 505: 344-352, 2014.

15. Prensner JR and Chinnaiyan AM: The emergence of lncRNAs in cancer biology. Cancer Discov 1: 391-407, 2011.

16. Hammond SM: An overview of microRNAs. Adv Drug Deliv Rev 87: 3-14, 2015. 
17. Ye W, Lv Q, Wong CK, Hu S, Fu C, Hua Z, Cai G, Li G, Yang BB and Zhang Y: The effect of central loops in miRNA: MRE duplexes on the efficiency of miRNA-mediated gene regulation. PLoS One 3: e1719, 2008.

18. Amirkhah R, Schmitz U, Linnebacher M, Wolkenhauer O and Farazmand A: MicroRNA-mRNA interactions in colorectal cancer and their role in tumor progression. Genes Chromosomes Cancer 54: 129-141, 2015.

19. Wang C, Hu DZ and Liu JZ: Identification of critical TF-miRNAmRNA regulation loops for colorectal cancer metastasis. Genet Mol Res 14: 5485-5495, 2015.

20. Salmena L, Poliseno L, Tay Y, Kats L and Pandolfi PP: A ceRNA hypothesis: The Rosetta Stone of a hidden RNA language? Cell 146: 353-358, 2011

21. Qi X, Zhang DH, Wu N, Xiao JH, Wang X and Ma W: ceRNA in cancer: Possible functions and clinical implications. J Med Genet 52: 710-718, 2015.

22. Militello G, Weirick T, John D, Döring C, Dimmeler S and Uchida S: Screening and validation of 1 ncRNAs and circRNAs as miRNA sponges. Brief Bioinform 18: 780-788, 2017.

23. Kallen AN, Zhou XB, Xu J, Qiao C, Ma J, Yan L, Lu L, Liu C, Yi JS, Zhang H, et al: The imprinted H19 lncRNA antagonizes let-7 microRNAs. Mol Cell 52: 101-112, 2013.

24. Imig J, Brunschweiger A, Brümmer A, Guennewig B, Mittal N, Kishore S, Tsikrika P, Gerber AP, Zavolan M and Hall J: miR-CLIP capture of a miRNA targetome uncovers a lincRNA H19-miR-106a interaction. Nat Chem Biol 11: 107-114, 2015.

25. Cesana M, Cacchiarelli D, Legnini I, Santini T, Sthandier O, Chinappi M, Tramontano A and Bozzoni I: A long noncoding RNA controls muscle differentiation by functioning as a competing endogenous RNA. Cell 147: 358-369, 2011.

26. Xia T, Liao Q, Jiang X, Shao Y, Xiao B, Xi Y and Guo J: Long noncoding RNA associated-competing endogenous RNAs in gastric cancer. Sci Rep 4: 6088, 2014

27. Zhang J, Fan D, Jian Z, Chen GG and Lai PB: Cancer specific long noncoding RNAs show differential expression patterns and competing endogenous RNA potential in hepatocellular carcinoma. PLoS One 10: e0141042, 2015.

28. Zhou X, Liu J and Wang W: Construction and investigation of breast-cancer-specific ceRNA network based on the mRNA and miRNA expression data. IET Syst Biol 8: 96-103, 2014.

29. Zhou M, Diao Z, Yue X, Chen Y, Zhao H, Cheng L and Sun J: Construction and analysis of dysregulated lncRNA-associated ceRNA network identified novel lncRNA biomarkers for early diagnosis of human pancreatic cancer. Oncotarget 7: 5638356394, 2016.

30. Wotschofsky Z, Gummlich L, Liep J, Stephan C, Kilic E, Jung K, Billaud JN and Meyer HA: Integrated microRNA and mRNA signature associated with the transition from the locally confined to the metastasized clear cell renal cell carcinoma exemplified by miR-146-5p. PLoS One 11: e0148746, 2016.

31. Fachel AA, Tahira AC, Vilella-Arias SA, Maracaja-Coutinho V, Gimba ER, Vignal GM, Campos FS, Reis EM and VerjovskiAlmeida S: Expression analysis and in silico characterization of intronic long noncoding RNAs in renal cell carcinoma: Emerging functional associations. Mol Cancer 12: 140, 2013.

32. Wang X, Chen X, Han W, Ruan A, Chen L, Wang R, Xu Z, Xiao P, Lu X, Zhao Y, et al: miR-200c targets CDK2 and suppresses tumorigenesis in renal cell carcinoma. Mol Cancer Res 13: 1567-1577, 2015.

33. Jeggari A, Marks DS and Larsson E: miRcode: A map of putative microRNA target sites in the long non-coding transcriptome. Bioinformatics 28: 2062-2063, 2012.

34. Hsu SD, Tseng YT, Shrestha S, Lin YL, Khaleel A, Chou CH, Chu CF, Huang HY, Lin CM, Ho SY, et al: miRTarBase update 2014: An information resource for experimentally validated miRNA-target interactions. Nucleic Acids Res 42: D78-D85, 2014.

35. Shannon P, Markiel A, Ozier O, Baliga NS, Wang JT, Ramage D, Amin N, Schwikowski B and Ideker T: Cytoscape: A software environment for integrated models of biomolecular interaction networks. Genome Res 13: 2498-2504, 2003.

36. Walsh AL, Tuzova AV, Bolton EM, Lynch TH and Perry AS: Long noncoding RNAs and prostate carcinogenesis: The missing 'linc'? Trends Mol Med 20: 428-436, 2014.
37. Zhang F, Zhang $\mathrm{L}$ and Zhang C: Long noncoding RNAs and tumorigenesis: Genetic associations, molecular mechanisms, and therapeutic strategies. Tumour Biol 37: 163-175, 2016.

38. Wang H, Niu L, Jiang S, Zhai J, Wang P, Kong F and Jin X: Comprehensive analysis of aberrantly expressed profiles of lncRNAs and miRNAs with associated ceRNA network in muscle-invasive bladder cancer. Oncotarget 7: 86174-86185, 2016.

39. Hauptman $\mathrm{N}$ and Glavač D: Long non-coding RNA in cancer. Int J Mol Sci 14: 4655-4669, 2013

40. Han D, Gao X, Wang M, Qiao Y, Xu Y, Yang J, Dong N, He J, Sun Q, Lv G, et al: Long noncoding RNA H19 indicates a poor prognosis of colorectal cancer and promotes tumor growth by recruiting and binding to eIF4A3. Oncotarget 7: 22159-22173, 2016.

41. Honda K: The biological role of actinin-4 (ACTN4) in malignant phenotypes of cancer. Cell Biosci 5: 41, 2015.

42. Honda K, Yamada T, Endo R, Ino Y, Gotoh M, Tsuda H, Yamada Y, Chiba H and Hirohashi S: Actinin-4, a novel actin-bundling protein associated with cell motility and cancer invasion. J Cell Biol 140: 1383-1393, 1998.

43. Yamamoto S, Tsuda H, Honda K, Onozato K, Takano M, Tamai S, Imoto I, Inazawa J, Yamada T and Matsubara O: Actinin-4 gene amplification in ovarian cancer: A candidate oncogene associated with poor patient prognosis and tumor chemoresistance. Mod Pathol 22: 499-507, 2009.

44. Kikuchi S, Honda K, Tsuda H, Hiraoka N, Imoto I, Kosuge T, Umaki T, Onozato K, Shitashige M, Yamaguchi U, et al: Expression and gene amplification of actinin-4 in invasive ductal carcinoma of the pancreas. Clin Cancer Res 14: 53485356, 2008.

45. Rojas AM, Fuentes G, Rausell A and Valencia A: The Ras protein superfamily: Evolutionary tree and role of conserved amino acids. J Cell Biol 196: 189-201, 2012.

46. Chen D, Guo J, Miki T, Tachibana M and Gahl WA: Molecular cloning of two novel rab genes from human melanocytes. Gene 174: 129-134, 1996.

47. Abba MC, Hu Y, Sun H, Drake JA, Gaddis S, Baggerly K, Sahin A and Aldaz CM: Gene expression signature of estrogen receptor alpha status in breast cancer. BMC Genomics 6: 37, 2005.

48. Luther T, Kotzsch M, Meye A, Langerholc T, Füssel S, Olbricht N, Albrecht S, Ockert D, Muehlenweg B, Friedrich K, et al: Identification of a novel urokinase receptor splice variant and its prognostic relevance in breast cancer. Thromb Haemost 89: 705-717, 2003.

49. Kotzsch M, Farthmann J, Meye A, Fuessel S, Baretton G, TjanHeijnen VC, Schmitt M, Luther T, Sweep FC, Magdolen V and Span PN: Prognostic relevance of uPAR-del4/5 and TIMP-3 mRNA expression levels in breast cancer. Eur J Cancer 41: 27602768,2005

50. Sato S, Kopitz C, Grismayer B, Beaufort N, Reuning U, Schmitt M, Luther T, Kotzsch M, Krüger A and Magdolen V: Overexpression of the urokinase receptor mRNA splice variant uPAR-del4/5 affects tumor-associated processes of breast cancer cells in vitro and in vivo. Breast Cancer Res Treat 127: 649-657, 2011.

51. Kunkle BW, Yoo C and Roy D: Reverse engineering of modified genes by Bayesian network analysis defines molecular determinants critical to the development of glioblastoma. PLoS One 8: e64140, 2013

52. Usó M, Jantus-Lewintre E, Sirera R, Bremnes RM and Camps C: miRNA detection methods and clinical implications in lung cancer. Future Oncol 10: 2279-2292, 2014.

53. Yoshino H, Seki N, Itesako T, Chiyomaru T, Nakagawa M and Enokida H: Aberrant expression of microRNAs in bladder cancer. Nat Rev Urol 10: 396-404, 2013.

54. Kumra H and Reinhardt DP: Fibronectin-targeted drug delivery in cancer. Adv Drug Deliv Rev 97: 101-110, 2016.

55. Muller PA and Vousden KH: Mutant p53 in cancer: New functions and therapeutic opportunities. Cancer Cell 25: 304-317, 2014.

56. Huang $\mathrm{H}$, Weng $\mathrm{H}$, Zhou $\mathrm{H}$ and $\mathrm{Qu} \mathrm{L}$ : Attacking c-Myc: Targeted and combined therapies for cancer. Curr Pharm Des 20: 6543-6554, 2014. 